\title{
Lipophilicity of the Cystic Fibrosis Drug, Ivacaftor (VX-770), and Its Destabilizing Effect on the Major CF-causing Mutation: F508del[S
}

\author{
Stephanie Chin, Maurita Hung, Amy Won, Yu-Sheng Wu, Saumel Ahmadi, Donghe Yang, \\ Salma Elmallah, Krimo Toutah, C. Michael Hamilton, Robert N. Young, Russell D. Viirre, \\ Christopher M. Yip, and Christine E. Bear \\ Department of Molecular Medicine, Hospital for Sick Children, Toronto, Ontario, Canada (S.C., M.H., Y.-S.W., S.A., D.Y., C.E.B.); \\ Departments of Biochemistry (S.C., C.E.B.) and Physiology (M.H., Y.-S.W., S.A., C.E.B.), and Institute of Biomaterials and \\ Biomedical Engineering (A.W., C.M.Y.), University of Toronto, Toronto, Ontario, Canada; Department of Chemistry and Biology, \\ Ryerson University, Toronto, Ontario, Canada (S.E., K.T., R.D.V.); and Department of Chemistry, Simon Fraser University, \\ Burnaby, British Columbia, Canada (C.M.H., R.N.Y.)
}

Received February 14, 2018; accepted June 13, 2018

\section{ABSTRACT}

Deletion of phenylalanine at position 508 (F508del) in cystic fibrosis transmembrane conductance regulator (CFTR) is the most common cystic fibrosis (CF)-causing mutation. Recently, ORKAMBI, a combination therapy that includes a corrector of the processing defect of F508del-CFTR (lumacaftor or VX-809) and a potentiator of channel activity (ivacaftor or VX-770), was approved for CF patients homozygous for this mutation. However, clinical studies revealed that the effect of ORKAMBI on lung function is modest and it was proposed that this modest effect relates to a negative impact of VX-770 on the stability of F508delCFTR. In the current studies, we showed that this negative effect of VX-770 at $10 \mu \mathrm{M}$ correlated with its inhibitory effect on VX-809-mediated correction of the interface between the second membrane spanning domain and the first nucleotide binding domain bearing F508del. Interestingly, we found that VX-770 exerted a similar negative effect on the stability of other membrane localized solute carriers (SLC26A3, SLC26A9, and SLC6A14), suggesting that this negative effect is not specific for F508del-CFTR. We determined that the relative destabilizing effect of a panel of VX-770 derivatives on F508del-CFTR correlated with their predicted lipophilicity. Polarized total internal reflection fluorescence microscopy on a supported lipid bilayer model shows that VX-770, and not its less lipophilic derivative, increased the fluidity of and reorganized the membrane. In summary, our findings show that there is a potential for nonspecific effects of VX-770 on the lipid bilayer and suggest that this effect may account for its destabilizing effect on VX-809- rescued F508del-CFTR.

\section{Introduction}

Cystic fibrosis (CF) is caused by mutations of a unique ATP-binding cassette anion channel, cystic fibrosis transmembrane conductance regulator (CFTR) (Cutting, 2015). CFTR has a lasso motif, two nucleotide binding domains (NBDs), two membrane spanning domains (MSDs), and a regulatory domain. In addition, the interface between the NBDs and MSDs consists of alpha-helical intracellular loops (ICLs) and coupling helices. The ICL:NBD1 interface is disrupted by the major CF-causing mutation, deletion of phenylalanine at position 508 (F508del), thereby compromising

This work was supported by Cystic Fibrosis Canada; Canadian Institutes of Health Research [Grants MOP-97954, GPG-102171]; and Natural Sciences and Engineering Research Council [Grant RGPIN-2015-043].

https://doi.org/10.1124/mol.118.112177.

S This article has supplemental material available at molpharm. aspetjournals.org. assembly and function of CFTR (Mendoza et al., 2012; Rabeh et al., 2012).

The first US Food and Drug Administration-approved medicine to treat the underlying cause of CF is KALYDECO (ivacaftor or VX-770), a potentiator that enhances channel gating of wild-type (wt) CFTR and CFTR with certain gating mutations (i.e., glycine to aspartic acid at position 551 or G551D) at the cell surface by directly binding to the protein to prolong its open state in an ATP-independent manner (Van Goor et al., 2009; Eckford et al., 2012; Jih and Hwang, 2013). On the other hand, patch clamp studies by Jih and Hwang (2013) suggested that both ATP-dependent and ATP-independent CFTR channel gating are modified by VX-770. Recently, ORKAMBI, a combination of VX-770 and a corrector compound known as lumacaftor (VX-809), was approved for use in patients who are homozygous for the major CF mutation, F508del. The corrector compound, VX-809, acts to partially rescue the defect in protein mistrafficking and misprocessing exhibited by F508del-CFTR

ABBREVIATIONS: BMOE, bismaleimidoethane; bSM, egg sphingomyelin; CF, cystic fibrosis; CFTR, cystic fibrosis transmembrane conductance regulator; DMEM, Dulbecco's modified Eagle's medium; DOPC, dioleoylphosphatidylcholine; Endo, endoglycosidase; F508del, deletion of phenylalanine at position 508; FBS, fetal bovine serum; Fsk, forskolin; HEK, human embryonic kidney; ICL, intracellular loop; logP, partition coefficient; MSD, membrane spanning domain; NBD, nucleotide binding domain; NEAA, nonessential amino acids; pTIRF, polarized total internal reflection fluorescence; SLC, solute carrier; wt, wild type. 
to the cell surface (Van Goor et al., 2011) by repairing the aberrant ICL:NBD1 interfaces (Rabeh et al., 2012; Loo et al., 2013; Okiyoneda et al., 2013; Ren et al., 2013). VX-770 acts on the VX-809-corrected F508del-CFTR to augment its activity (Van Goor et al., 2009, 2011; Eckford et al., 2012; Jih and Hwang, 2013).

Unfortunately, ORKAMBI mediates modest and variable clinical responses (Boyle et al., 2014), prompting further discovery efforts for more efficacious corrector compounds. Certain in vitro findings suggest that the modest in vivo effect may reflect an adverse effect of VX-770 on the stability of the VX-809-corrected protein (Cholon et al., 2014; Veit et al., 2014; Matthes et al., 2016). Thus, the aim of the current studies was to interrogate the mechanism underlying this destabilizing effect of VX-770 on the major CF mutant, F508del. We also assessed the specificity of this destabilizing effect of VX-770 by studying its interaction with other membrane proteins (including SLC26A3, SLC26A9, and SLC6A14) as well as the lipid bilayer.

\section{Materials and Methods}

Chronic Treatment of F508del-CFTR with VX-770 and Its Analogs. Human embryonic kidney (HEK)-293 cells stably expressing F508del-CFTR (HEK F508del) were plated on 24-well plates (Sarstedt, Nümbrecht, Germany) and maintained in Dulbecco's Modified Eagle's Medium (DMEM; Wisent, St-Bruno, Quebec, Canada) supplemented with $10 \%$ Fetal Bovine Serum (FBS; Wisent), 1\% nonessential amino acids (NEAA; Wisent), $0.6 \mathrm{mg} / \mathrm{ml}$ G418 sulfate (Wisent), and $5 \mu \mathrm{g} / \mathrm{ml}$ blasticidin (Wisent) at $37^{\circ} \mathrm{C}$ with $5 \% \mathrm{CO}_{2}$ overnight. For the initial chronic VX-770 studies, HEK F508del cells were pretreated with VX-770 at a pharmacological concentration $(0.1 \mu \mathrm{M})$ or at a suprapharmacological concentration $(10 \mu \mathrm{M})$ with $3 \mu \mathrm{M}$ VX-809 (Selleck Chemicals, Houston, TX) in the previously described media at $37^{\circ} \mathrm{C}$ with $5 \% \mathrm{CO}_{2}$ for 48 hours. For the studies with VX-770 and its derivatives, HEK F508del cells were pretreated with VX-770 (Selleck Chemicals) or its various derivatives (SE and KT compounds) synthesized in Dr. Russell Viirre's laboratory (Toronto, Ontario, Canada) (Krimo Toutah, Maurita Hung, Salma Elmallah, C. Michael Hamilton, Robert N. Young, Christine E. Bear and Russell D. Viirre, manuscript in preparation, refer to Supplemental Fig. 2 for chemical structures of VX-770 derivatives) at a pharmacological concentration $(0.1 \mu \mathrm{M})$ or at a suprapharmacological concentration $(10 \mu \mathrm{M})$ in the previously described media at $27^{\circ} \mathrm{C}$ with $5 \% \mathrm{CO}_{2}$ for 24 hours. The synthesis and characterization of the SE compounds in this paper have been described previously in detail (http://digital. library.ryerson.ca/islandora/object/RULA:1478). The synthesis and characterization of the KT compounds employed the same methodology as (http://digital.library.ryerson.ca/islandora/object/RULA:1478) for the most part but also incorporated additional reaction steps previously published by the Verkman group for modification of the left-hand side of the VX-770 molecule (Pedemonte et al., 2005).

Generation of Mutants and Constructs. The V510C, A1067C, and V510C/A1067C cysteine-less (cys-less) variants of wt-CFTR were kindly provided by Dr. David Clarke (Toronto, Ontario, Canada); the SLC26A3 and SLC26A9 constructs with N-terminal FLAG tags were kindly provided by Dr. Reinhart Reithmeier (Toronto, Ontario, Canada); and the SLC6A14 construct with a C-terminal FLAG tag was generated by OriGene (Rockville, MD). The F508del mutation was generated on the V510C/A1067C cys-less variant of wt-CFTR background with primers (forward: 5' -CATTAAAGAAAATATCATTGGTTGTTCCTATGATG-3' and reverse: 5'-CATCATAGGAACAACCAATGATATTTTCTTTAATG- ${ }^{\prime}$ ) and the KAPA HiFi HotStart PCR Kit (KAPA Biosystems, Woburn, MA). Plasmid DNA was expanded with the QIAprep Spin Miniprep Kit (Qiagen, Hilden, Germany), and the sequence was confirmed with DNA sequencing (TCAG, Toronto, Ontario, Canada). HEK-293 GripTite (GT) cells, kindly provided by Dr. Daniella Rotin's laboratory (Toronto, Ontario, Canada), were maintained in DMEM (Wisent) supplemented with $10 \%$ FBS (Wisent), 1\% NEAA (Wisent), and $0.6 \mathrm{mg} / \mathrm{ml} \mathrm{G418}$ sulfate (Wisent), transfected with constructs using PolyFect Transfection Reagent (Qiagen) according to the manufacturer's protocol and temperature-rescued at $27^{\circ} \mathrm{C}$ with $5 \% \mathrm{CO}_{2}$ for 24 hours.

Cysteine Cross-linking. HEK-293 GT cells were grown on 24-well plates (Sarstedt) at $37^{\circ} \mathrm{C}$ with $5 \% \mathrm{CO}_{2}$ overnight and then transfected with cys-less variants of CFTR. Cells were treated with DMSO $(0.1 \%)$, VX-809 $(3 \mu \mathrm{M})$, VX-770 $(0.1$ or $10 \mu \mathrm{M})$, or VX-809 plus VX-770 $(0.1$ or $10 \mu \mathrm{M})$ at $27^{\circ} \mathrm{C}$ with $5 \% \mathrm{CO}_{2}$ for 24 hours. The next day, cells were treated with $50 \mu \mathrm{M}$ bismaleimidoethane (BMOE; Life Technologies, Rockford, IL) in DMEM (Wisent) supplemented with $10 \%$ FBS (Wisent), 1\% NEAA (Wisent), and $0.6 \mathrm{mg} / \mathrm{ml} \mathrm{G418} \mathrm{sulfate}$ (Wisent) at $27^{\circ} \mathrm{C}$ with $5 \% \mathrm{CO}_{2}$ for 1 hour.

Deglycosylation Studies. Cys-less variants of CFTR were lysed from HEK-293 GT cells into radioimmunoprecipitation assay [50 mM Tris-Base, $150 \mathrm{mM}$ sodium chloride, $1 \mathrm{mM}$ EDTA, 1\% (v/v) Triton $\mathrm{X}-100,0.1 \%(\mathrm{v} / \mathrm{v})$ SDS, $1 \times$ protease inhibitor cocktail (AMRESCO, Cleveland, $\mathrm{OH}$ ), pH 7.4] buffer. Protein samples were treated with Endoglycosidase $\mathrm{H}$ and PNGase F according to the manufacturer's protocol (New England Biolabs, Ipswich, MA)

Chronic Treatment of SLC Proteins with VX-770. HEK-293 GT cells were grown on 24 -well plates (Sarstedt) at $37^{\circ} \mathrm{C}$ with $5 \% \mathrm{CO}_{2}$ overnight and transfected with the SLC constructs. Cells were then treated with various concentrations of VX-770 $(0,0.1,1,2,10 \mu \mathrm{M})$ at $27^{\circ} \mathrm{C}$ with $5 \% \mathrm{CO}_{2}$ for 24 hours.

Immunoblotting. HEK F508del and HEK-293 GT cells were lysed into radioimmunoprecipitation buffer and protein samples were subjected to SDS-PAGE. CFTR protein samples were run on 6\% Trisglycine sodium dodecyl sulfate (TG SDS) gels (Life Technologies, Carlsbad, CA) and SLC protein samples were run on 8\% TG SDS gels (Life Technologies). Protein samples were transferred to nitrocellulose paper and probed with the following primary antibodies overnight at $4^{\circ} \mathrm{C}$ shaking: IgG2b mAb596 antibody (University of North Carolina at Chapel Hill, Chapel Hill, NC, code: A4; Cystic Fibrosis Foundation Therapeutics Inc., Bethesda, MD) for CFTR protein (1:5000 for F508del-CFTR, 1:1000 for V510C/A1067C cysless variant of wt-CFTR, 1:500 for V510C/A1067C cys-less variant of F508del-CFTR), calnexin-specific rabbit pAb (1:10,000, cat. no.: C4731; Sigma-Aldrich, St. Louis, MO) for calnexin loading control, anti-mouse FLAG antibody (1:2500; Sigma-Aldrich) for SLC protein, and anti$\beta$-actin (1:10,000, ab8229; Abcam, Cambridge, UK) for actin loading control. The samples were then probed with secondary antibodies for 1 hour at room temperature shaking at concentrations twice as diluted as the primary antibodies as follows: horseradish peroxidaseconjugated goat anti-mouse IgG secondary antibody (Pierce, Rockford, IL) for CFTR protein and actin loading control, horseradish peroxidase-conjugated goat anti-rabbit IgG secondary antibody (Pierce) for calnexin loading control, and anti-mouse HA (MMS101R; Covance, Princeton, NJ) for SLC protein. Immunoblots were exposed using Amersham enhanced chemiluminescent reagent (GE Healthcare Life Sciences, Mississauga, Ontario, Canada) with the Li-Cor Odyssey Fc (LI-COR Biosciences, Lincoln, NE) at a linear range of exposure. Densitometry of immunoblot bands were analyzed with Image Studio Lite software (Version 5.2.5) from Li-Cor Odyssey Fc (LI-COR Biosciences).

FLiPR Membrane Potential Assay. The FLiPR membrane potential assay was conducted as previously described (Molinski et al., 2015). Briefly, HEK F508del cells were grown on black 96-well plates with clear bottoms (Corning, Corning, NY) at $37^{\circ} \mathrm{C}$ with $5 \% \mathrm{CO}_{2}$ overnight. Cells were then treated with $3 \mu \mathrm{M}$ VX- 809 at $37^{\circ} \mathrm{C}$ with $5 \%$ $\mathrm{CO}_{2}$ for 48 hours. The next day, cells were loaded with $0.5 \mathrm{mg} / \mathrm{ml}$ FLiPR membrane potential dye (Molecular Devices, Sunnyvale, CA) in sodium gluconate buffer as previously described (Molinski et al., 2015) for 45 minutes with $5 \% \mathrm{CO}_{2}$ at $37^{\circ} \mathrm{C}$. Fluorescence was read at excitation of $530 \mathrm{~nm}$ and emission of $560 \mathrm{~nm}$ on the SpectraMax i3X 
fluorescence plate reader at $37^{\circ} \mathrm{C}$ (Molecular Devices). Baseline fluorescence was read for 5 minutes. CFTR was stimulated by vehicle, $1 \mu \mathrm{M}$ forskolin (Fsk; Sigma-Aldrich), $1 \mu \mathrm{M}$ Fsk with VX-770 $(10 \mu \mathrm{M})$, or $1 \mu \mathrm{M}$ Fsk with the derivative SE-03 $(10 \mu \mathrm{M})$ for 10 minutes. CFTR was then inhibited with $10 \mu \mathrm{M}$ CFTR inhibitor-172 $\left(\mathrm{CFTR}_{\mathrm{inh}^{-172}}\right.$; Cystic Fibrosis Foundation Therapeutics Inc. and Rosalind Franklin University of Medicine and Science, Chicago, IL) for 12 minutes. Curves were normalized to last point of baseline and the maximal points of stimulation were plotted with GraphPad Prism v6.01 (San Diego, CA).

Polarized Total Internal Reflection Fluorescence Microscopy. The formation of supported lipid bilayers of 1:1:1 dioleoylphosphatidylcholine/egg sphingomyelin/cholesterol (DOPC/bSM/cholesterol) and 1:1:1 DOPC/distearoylphosphatidylcholine/cholesterol with DilC $_{18}(3)$ (1 mol \%; Life Technologies) in buffer (10 mM HEPES, $150 \mathrm{mM} \mathrm{NaCl}, \mathrm{pH}$ 7.4) were prepared as previously described (Walsh et al., 2014). All images were acquired using a home-built pTIRF system incorporating four excitation laser lines built on an Olympus IX70 inverted microscope. All images were captured at 15-second intervals for 1 hour with a water-cooled Evolve 512 EMCCD camera (Photometrics, Tucson, AZ) controlled by $\mu$-Manager (http://www.micro-manager.org). Fluorescent probes were excited by substrate parallel ( $\mathrm{F} s, s$-polarized) and perpendicular ( $\mathrm{F} p, p$-polarized) polarized excitation at $532 \mathrm{~nm}$ through a half-wave liquid crystal variable retarder (LCC25 1111A; Thorlabs, Newton, NJ). Two bilayer studies were performed with multiple fields of view examined for each bilayer (three to six fields). Order parameter $<$ $\mathrm{P}_{2}>$ values were determined for each region of interest and calculated on a per pixel basis using an in-house macro. Refer to previous publications for more details (Oreopoulos and Yip, 2009; Oreopoulos et al., 2010).

Statistical Analyses. Results were plotted and analyzed on GraphPad Prism v6.01. Results were presented as mean \pm S.D. with each replicate representing one biological replicate from an individual experiment. For all statistical analyses, one-way ANOVA and Dunnett's post hoc test were conducted on GraphPad Prism v6.01 for multiple comparisons of conditions to the control. The $P$ values were automatically adjusted by GraphPad Prism v6.01 for the multiple comparisons and $P<0.05$ was considered significantly different from the control.

\section{Results}

Destabilizing Effect of Long-Term Treatment with Micromolar Concentration of VX-770 on the Abundance of VX-809-corrected F508del-CFTR Protein. As previously reported (Cholon et al., 2014; Veit et al., 2014; Matthes et al., 2016), we observed a destabilizing effect of long-term (48 hours) treatment of VX-770 at a high concentration $(10 \mu \mathrm{M})$ at $37^{\circ} \mathrm{C}$ on the relative abundance of the mature band C (Fig. 1B) and immature band B (Fig. 1C) forms of the VX-809-rescued F508del-CFTR protein in the HEK-293 expression system. As previously reported (Cholon et al., 2014), this was not related to a significant decrease in cell viability, with comparable abundance of calnexin expression across the treatments (Fig. 1A).

Micromolar Concentration of VX-770 Reverses the Effect of VX-809 in Stabilizing the ICL4:NBD1 Interface of Cys-Less Variant of F508del-CFTR. We were prompted to determine if this destabilizing effect of VX-770 was due, in part, to a reversal of the positive effect of VX-809 on F508delCFTR assembly. The corrector, VX-809, is thought to act by stabilizing the aberrant interaction between F508del in NBD1 and the intracellular loop ICL4 (conferred by MSD2) (Rabeh et al., 2012; Okiyoneda et al., 2013). As in previous publications (He et al., 2008; Loo et al., 2008; Serohijos et al., 2008; Aleksandrov et al., 2010; Loo and Clarke, 2011; Chin et al., 2017), we assessed this interaction by studying the propensity for chemical cross-linking between cysteine residues engineered at V510 (NBD1) and A1067 (ICL4) in the context of a full-length cysteine-less (cys-less) variant of CFTR protein for both wt and F508del. The following studies were performed at $27^{\circ} \mathrm{C}$ as the cys-less variant of CFTR protein is misprocessed at $37^{\circ} \mathrm{C}$ and requires temperature rescue at $27^{\circ} \mathrm{C}$ to promote its maturation (Loo and Clarke, 2006; Wang et al., 2007; He et al., 2008, 2013).

The V510C/A1067C cys-less variant of wt-CFTR runs as two bands: the mature, complex glycosylated band $\mathrm{C}$ and the immature, core glycosylated band B (Fig. 2A). As previously reported, band $\mathrm{C}$ is insensitive to Endoglycosidase (Endo) $\mathrm{H}$, whereas band B is deglycosylated, and this form is labeled as band $\mathrm{A}$. Both band $\mathrm{B}$ and $\mathrm{C}$ are deglycosylated by PNGase $\mathrm{F}$ as shown on the left panels of Fig. 2A. We confirmed that two cysteine residues (V510C on NBD1 and A1067C on ICL4) could be cross-linked by a cell-permeable maleimide reagent, bismaleimidoethane (BMOE), in a V510C/A1067C cys-less variant of wt-CFTR protein with the appearance of a crosslinked species ( $\boldsymbol{t}$ ) that migrates as a $250-\mathrm{kDa}$ protein on a SDSPAGE gel (Fig. 2A) (Chin et al., 2017). We confirmed that this band (t) was conferred by intramolecular rather than intermolecular cross-linking, because it was not produced when single cysteine-containing constructs (V510C or A1067C) were expressed alone or together (Supplemental Fig. 1) (Chin et al., 2017). We showed that the band (t) is Endo H resistant and, hence, complex glycosylated. Interestingly, an $\mathrm{N}$-glycanase resistant band $(\triangle)$ was apparent in the cross-linker-treated cells but not in the absence of cross-linker (Fig. 2A; Supplemental Fig. 1). We suggest on the basis of its glycosidase resistance that this unique band is the cross-linked form of band $\mathrm{A}$.

A V510C/A1067C cys-less variant of F508del-CFTR is misprocessed with no appearance of band $\mathrm{C}$ on the immunoblot, with just the misprocessed band $\mathrm{B}$ expressed (Fig. 2A) (Loo et al., 2008; Loo and Clarke, 2011). Treatment with the cell-permeable cross-linker (BMOE) led to the appearance of a unique band (*). This band (*) shifted slightly following Endo $\mathrm{H}$ treatment to band ( $\triangle$ ), confirming that it is not the $\mathrm{C}$ band, but rather the cross-linked band $\mathrm{B}$. The band $(\triangle)$ of this mutant protein is $\mathrm{N}$-glycanase resistant, suggesting that it represents the cross-linked band A.

Long-term (24 hours) treatment of V510C/A1067C cys-less variant of F508del-CFTR with VX-809 $(3 \mu \mathrm{M})$ did not lead to its maturation and the appearance of band C, likely due to the number of mutations introduced to eliminate endogenous cysteines (Fig. 2B). However, this treatment did lead to a significant increase in the cross-linking between V510C and A1067C with an increased ratio of the cross-linked band B $(*)$ relative to band $\mathrm{B}$ (Fig. 2, $\mathrm{B}$ and $\mathrm{C}$ ). These findings are consistent with previous studies, showing its positive effect in improving interaction between NBD1 and ICL4 (Van Goor et al., 2011; Loo et al., 2013; Ren et al., 2013). This positive effect of VX-809 was reversed when VX-770 $(10 \mu \mathrm{M})$ was included in this long-term treatment (Fig. 2, B and C). This abolition of NBD1:ICL4 assembly following the combination treatment could explain the effect observed on processing as documented in Fig. 1. As in the case of the protein processing studies shown in Fig. 1, the destabilizing effect of VX-770 on domain:domain assembly is dose dependent, and no destabilizing effect on cross-linking was observed following cotreatment with VX-770 at $0.1 \mu \mathrm{M}$ (Fig. 2, B and C). 
A

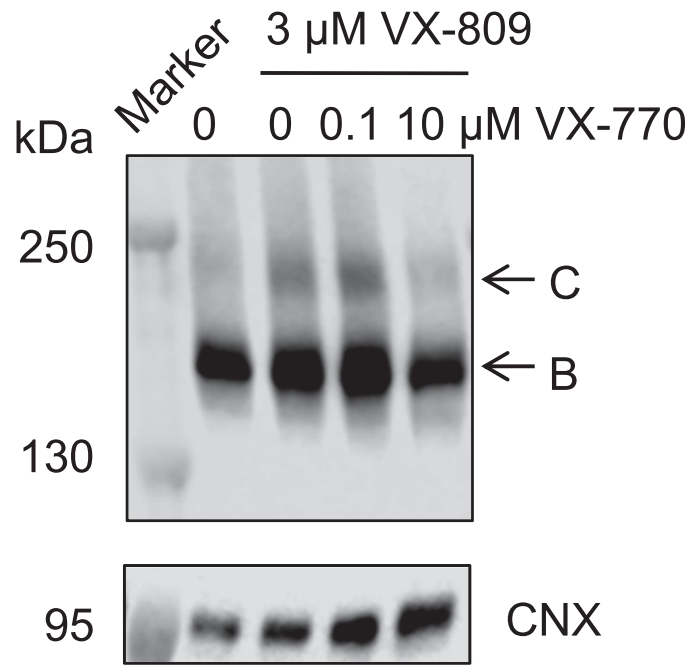

B

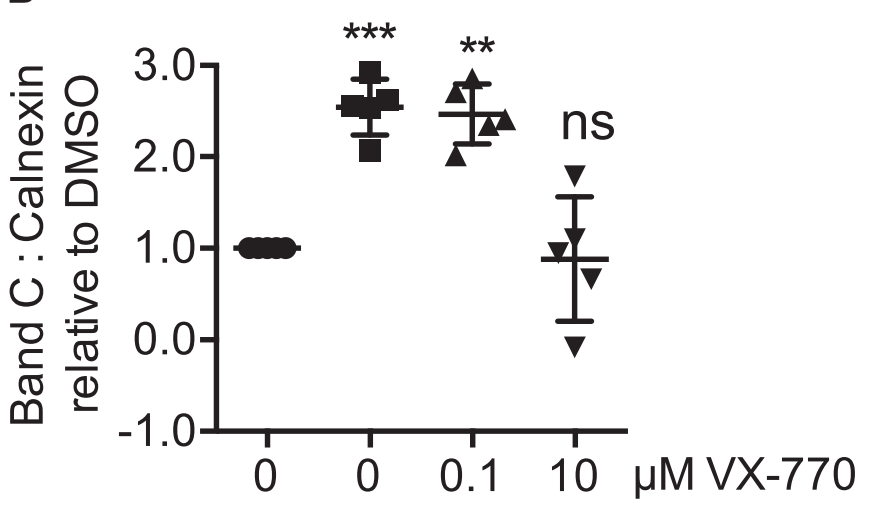

$3 \mu \mathrm{MVX}-809$

C

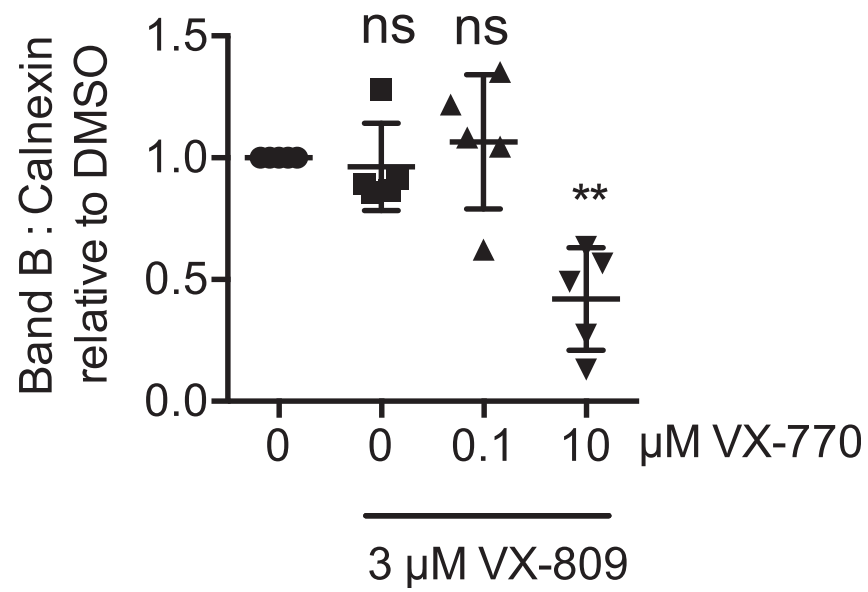

Fig. 1. Suprapharmacological concentration of VX-770 destabilizes protein expression of VX-809-rescued F508del-CFTR. (A) Immunoblot of F508del-CFTR treated with $3 \mu \mathrm{M}$ VX-809 and 0, 0.1, and $10 \mu \mathrm{M}$ VX-770 at $37^{\circ} \mathrm{C}$ for 48 hours with no VX-809 treatment as control and calnexin (CNX) as loading control. (B) Densitometry analysis of immunoblots of
Micromolar Concentrations of VX-770 Also Exert a Destabilizing Impact on Other Membrane Proteins. We were interested to determine if the destabilizing effect of $10 \mu \mathrm{M}$ VX-770 was specific to F508del-CFTR. To maintain similar conditions to our cysteine cross-linking studies, we tested its specificity by applying this concentration of VX-770 at $27^{\circ} \mathrm{C}$ on HEK-293 cells expressing other membrane proteins, including solute carriers (SLCs): SLC26A3, SLC26A9, and SLC6A14. These membrane proteins are particularly important, because they have been shown to interact with and/or modify CFTR channel activity in various epithelial tissues (Ko et al., 2004; Xu et al., 2005; Gupta et al., 2006; Bertrand et al., 2009; Chang et al., 2009; Avella et al., 2011; Karunakaran et al., 2011; Anagnostopoulou et al., 2012). Furthermore, these SLC genes have been shown to modify CF disease phenotypes (Sun et al., 2012; Li et al., 2014b; Corvol et al., 2015; Strug et al., 2016). Thus we reason that it would be disadvantageous for CFTR modulatory drugs to also affect the expression of these membrane proteins.

Firstly, analysis of the SLC proteins by SDS-PAGE revealed multiple glycosylation states as previously reported (Li et al., 2014a). Interestingly, the steady-state abundance of all the SLCs in this study was significantly decreased with chronic treatment with micromolar concentrations of VX-770 at $27^{\circ} \mathrm{C}$ (Fig. 3). SLC26A3 appeared to be the most susceptible to VX-770, because its protein expression significantly decreased at concentrations of VX-770 as low as $0.1 \mu \mathrm{M}$ (Fig. 3A). On the other hand, the abundance of SLC26A9 and SLC6A14 was significantly decreased starting at higher concentrations of 2 and $10 \mu \mathrm{M}$ VX-770 respectively (Fig. 3, B and C). Therefore, the destabilizing effect of suprapharmacological concentrations of VX-770 is not specific to F508del-CFTR.

Lipophilicity of VX-770 Appears To Be Correlated with the Destabilizing Effect on F508del-CFTR. We propose that the nonspecific, destabilizing effects of suprapharmacological concentrations of VX-770 may be related to the known effects of this compound on the lipid bilayer (Baroni et al., 2014). To test this idea, VX-770 derivatives (Supplemental Fig. 2) with varying degrees of lipophilicity, as assessed by their partition coefficient $(\log P)$ values, were incubated with HEK-293 cells expressing temperaturerescued F508del-CFTR and their effects on the processing (band $\mathrm{C} / \mathrm{C}+\mathrm{B}$ ) of F508del-CFTR determined.

In this study, the HEK-293 cells expressing F508del-CFTR were treated with VX-770 or a derivative for 24 hours at 0.1 or $10 \mu \mathrm{M}$ at $27^{\circ} \mathrm{C}$. We observed a negative correlation between the lipophilicity or $\log P$ values and the relative band $\mathrm{C} / \mathrm{C}+\mathrm{B}$ ratio (Fig. 4B). Derivatives with $\operatorname{low} \log P$ scores (i.e., SE-02, SE-03, KT-226, and KT-227) were associated with a higher

band $\mathrm{C}$ abundance normalized to calnexin (CNX) loading control and DMSO treatment shows that chronic treatment with $10 \mu \mathrm{M}$ VX-770, and not $0.1 \mu \mathrm{M}$ VX-770, significantly decreased band C abundance relative to VX-809 rescue of F508del-CFTR to a similar level as no VX-809 rescue $(n=5$ for all conditions). Results are presented as mean \pm S.D. and analyzed by one-way ANOVA and Dunnett's post hoc test (ns, not significant; $* * P<0.01$; $* * * P<$ 0.001 compared with vehicle). (C) Densitometry analysis of immunoblots of band B abundance normalized to calnexin (CNX) loading control and DMSO treatment shows that chronic treatment with $10 \mu \mathrm{M}$ VX-770, and not $0.1 \mu \mathrm{M}$ VX-770, also significantly decreased band $\mathrm{B}$ abundance relative to VX-809 rescue of F508del-CFTR ( $n=5$ for all conditions). Results are presented as mean \pm S.D. and analyzed by one-way ANOVA and Dunnett's post hoc test (ns, not significant; $* * P<0.01$ compared to vehicle). 

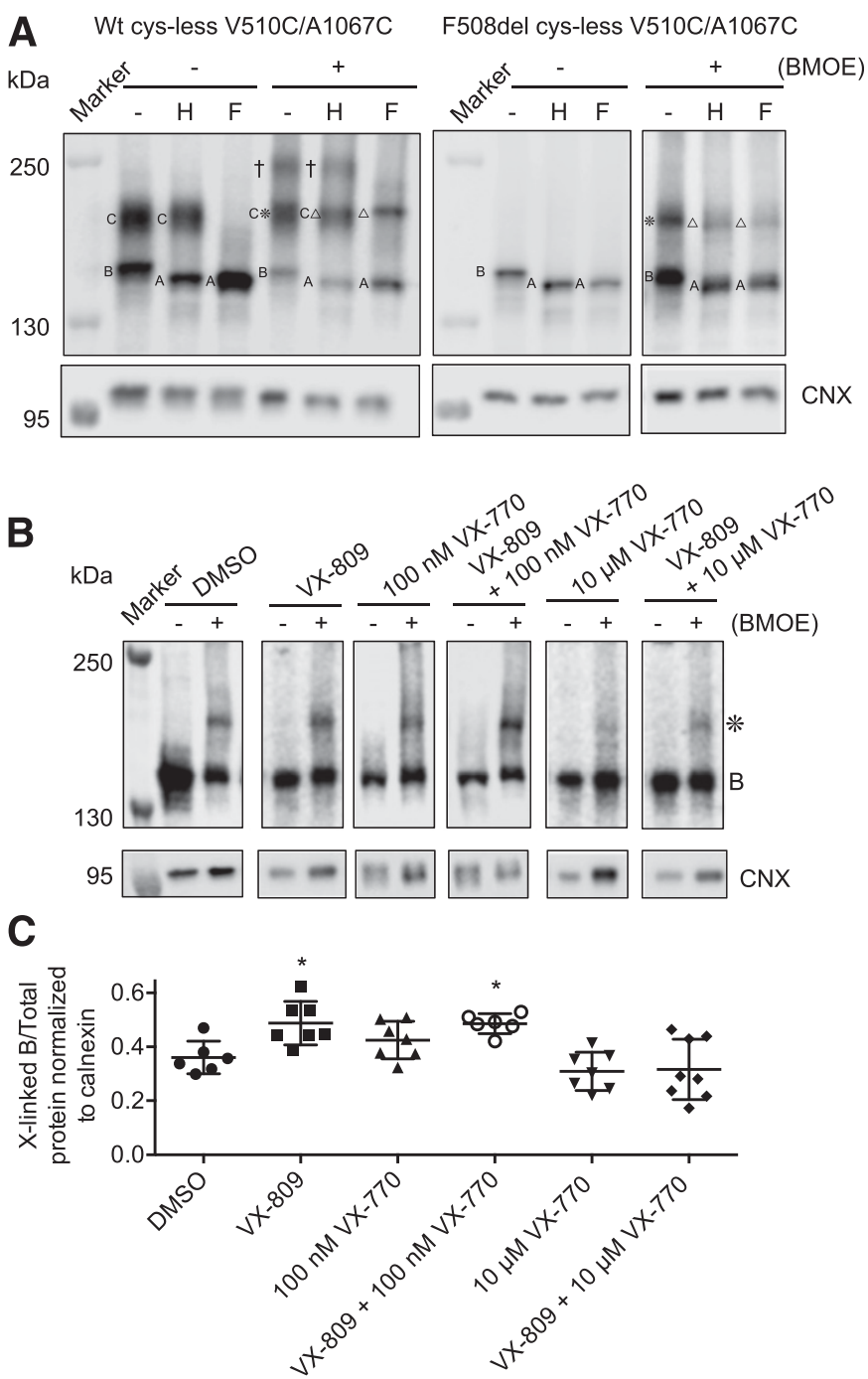

Fig. 2. Suprapharmacological concentration of VX-770 abolishes VX-809 rescue effect at the ICL4:NBD1 interface of cys-less variant of F508delCFTR. (A) Immunoblot of deglycosylation studies of V510C/A1067C cysless variants of wt- and F508del-CFTR without (-) and with (+) BMOE treatment. V510C/A1067C cys-less variant of wt-CFTR (-) BMOE: No glycanase (-) treatment resulted in bands $\mathrm{C}$ and B. Endoglycosidase $\mathrm{H}(\mathrm{H})$ treatment resulted in band $\mathrm{C}$ and band $\mathrm{A}$. PNGase $\mathrm{F}(\mathrm{F})$ treatment resulted in band A. V510C/A1067C cys-less variant of wt-CFTR (+) BMOE: No glycanase (-) treatment resulted in cross-linked band $\mathrm{C}(\mathbf{t})$, population containing band $\mathrm{C}$ and cross-linked band $\mathrm{B}\left(\mathrm{C}^{*}\right)$, and band B. Endoglycosidase $\mathrm{H}(\mathrm{H})$ treatment resulted in cross-linked band $\mathrm{C}(\mathbf{t})$, population of band $\mathrm{C}$ and cross-linked band $\mathrm{A}(\mathrm{C} \triangle \mathrm{)}$, and band $\mathrm{A}$. PNGase $\mathrm{F}(\mathrm{F})$ treatment resulted in cross-linked band $\mathrm{A}(\triangle)$, and band A. V510C/A1067C cys-less variant of F508del-CFTR (-) BMOE: No glycanase (-) treatment resulted in band $\mathrm{B}$, Endoglycosidase $\mathrm{H}(\mathrm{H})$ treatment resulted in band $\mathrm{A}$ and PNGase F (F) treatment resulted in band A. V510C/A1067C cys-less variant of F508del-CFTR (+) BMOE: No glycanase (-) treatment resulted in cross-linked band B $(*)$ and band B. Endoglycosidase $\mathrm{H}(\mathrm{H})$ treatment resulted in cross-linked band $A(\triangle)$ and band A. PNGase $F(F)$ treatment resulted in cross-linked band $\mathrm{A}(\triangle)$ and band $\mathrm{A}$. (B) Immunoblot of V510C/A1067C cys-less variant of F508del-CFTR pretreated with vehicle (DMSO, 0.1\%), VX-809 (3 $\mu \mathrm{M})$, VX-770 (100 nM or $10 \mu \mathrm{M})$, or combination of VX-809 and (+) VX-770 $(100 \mathrm{nM}$ VX-770 or $10 \mu \mathrm{M})$ at $27^{\circ} \mathrm{C}$ for 24 hours. (C) Densitometry results of normalized cross (X)-linked band B $(*)$ to band $\mathrm{B}$ show that VX-809 significantly increased the abundance of relative $\mathrm{X}$-linked band $\mathrm{B}(*)$, and this effect was abolished upon additional treatment with $10 \mu \mathrm{M}$ VX-770 but not with $100 \mathrm{nM}$ VX-770 $(n=6$ for DMSO and VX-809 + $100 \mathrm{nM}$ VX-770 conditions; $n=7$ for VX-809, $100 \mathrm{nM}$ VX-770 and $10 \mu \mathrm{M}$ VX-770 conditions; and $n=8$ for VX-809 $+10 \mu \mathrm{M}$ VX-770 condition). Results are presented as mean \pm S.D. and analyzed by one-way ANOVA and Dunnett's post hoc test $(* P<0.05$ compared with vehicle).


Fig. 3. VX-770 exerts destabilizing effect on membrane proteins of the solute carrier (SLC) family. (i) Representative immunoblots of (A) SLC26A3, (B) SLC26A9, and (C) SLC6A14 show different glycosylation forms of the proteins as previously reported ( $\mathrm{Li}$ et al., 2014a). SLC proteins were treated with $0,0.1,1,2$, and $10 \mu \mathrm{M}$ of VX-770 at $27^{\circ} \mathrm{C}$ for 24 hours with actin as loading control. (ii) Densitometry analyses of total protein relative to DMSO treatment and actin loading control of (A) SLC26A3 ( $n=$ 4 for all conditions), (B) SLC26A9 ( $n=5$ for all conditions), and (C) SLC6A14 ( $n=4$ for all conditions). SLC26A3 expression significantly decreased after $0.1 \mu \mathrm{M}$ VX-770, SLC26A9 expression significantly decreased after $2 \mu \mathrm{M}$ VX-770, and SLC6A14 expression significantly decreased after $10 \mu \mathrm{M}$ VX-770. Results are presented as mean \pm S.D. and analyzed by one-way ANOVA and Dunnett's post hoc test $(* P<0.05$; ** $P<$ $0.01 ; * * * P<0.001$ compared with vehicle).

band $\mathrm{C} / \mathrm{C}+\mathrm{B}$ ratio than the derivatives with high $\log P$ scores (i.e., KT-51, KT-126, KT-127, and KT-128) (Fig. 4, A and B). For example, the derivative SE-03, while exhibiting potentiator activity (Fig. 4, D and E), did not reduce steady-state protein abundance at $10 \mu \mathrm{M}$ (Fig. 4C).

Micromolar Concentration of VX-770 Increase the Membrane Fluidity in a Lipid Raft Model. Given the potential role for lipophilicity in VX-770-dependent effects on membrane protein stability, we were prompted to determine if the nonspecific destabilizing effects of micromolar concentrations of VX-770 were mediated by disrupting lipid rafts. We tested this hypothesis using a synthetic lipid raft model and tested the effect of micromolar VX-770 on membrane fluidity and bilayer integrity. Polarized total internal reflection fluorescence (pTIRF) microscopy was employed to study the effect of $10 \mu \mathrm{M}$ VX-770 on supported lipid bilayers that contained DOPC/cholesterol/distearoylphosphatidylcholine 

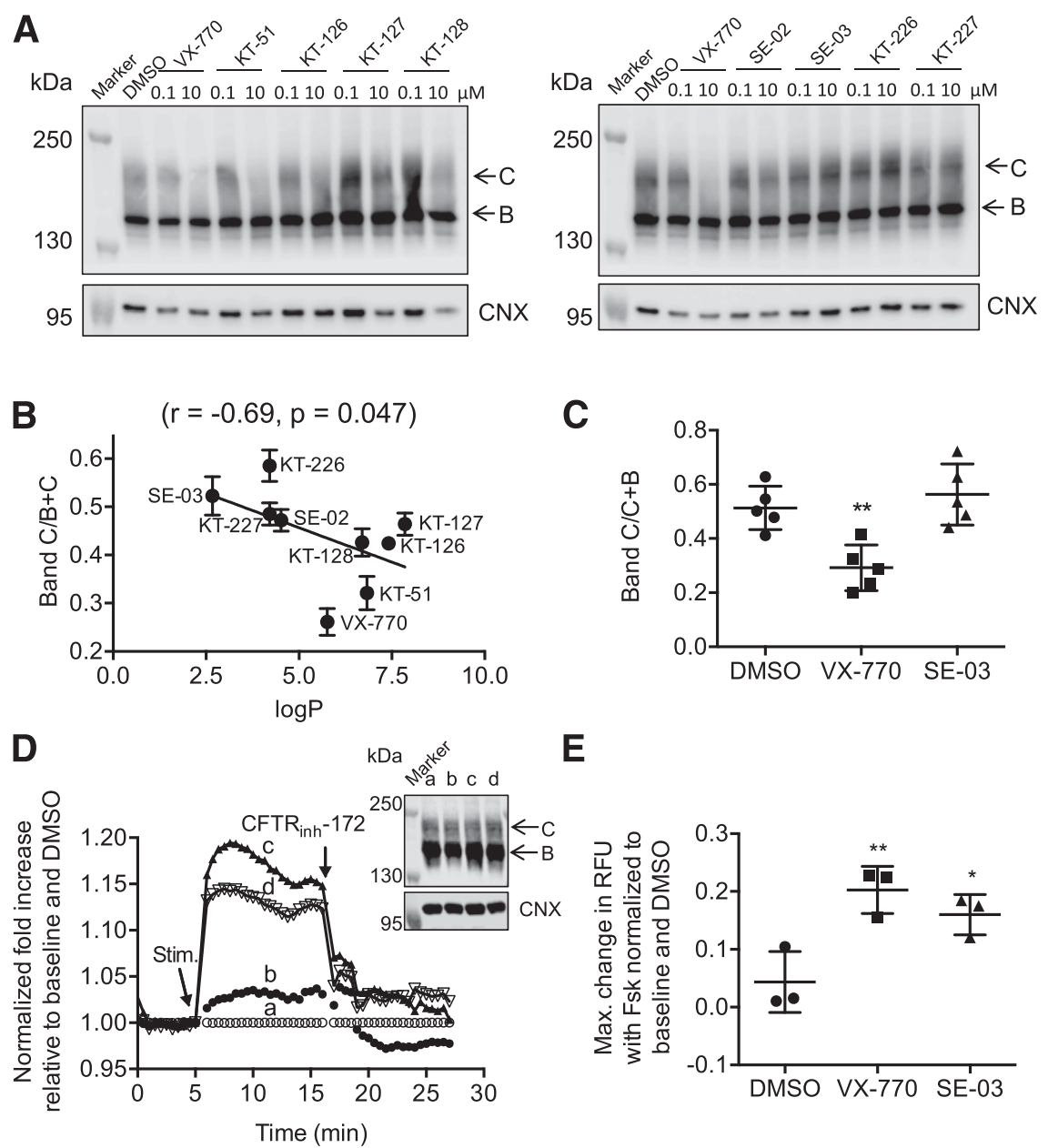

E

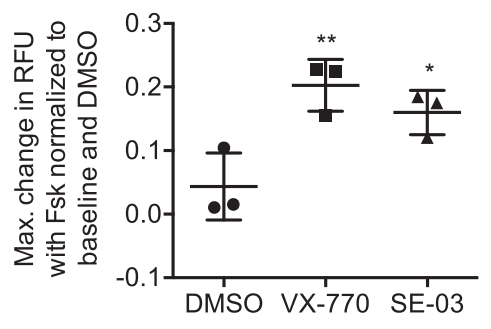

Fig. 4. Lipophilicity of VX-770 appears to correlate with its destabilizing effect on F508del-CFTR. (A) Immunoblots of F508del-CFTR from HEK-293 cells overexpressing F508del-CFTR chronically treated with VX-770 and its derivatives at 0.1 or $10 \mu \mathrm{M}$ at $27^{\circ} \mathrm{C}$ for 24 hours compared with DMSO as a negative control. Calnexin (CNX) was used as a loading control. (B) Correlation plot of the lipophilicity measure also known as $\log P$ and the abundance of cell surface band $\mathrm{C}$ relative to band $\mathrm{C}$ and band $\mathrm{B}(\mathrm{C}+\mathrm{B})$ of F508del-CFTR treated with $10 \mu \mathrm{M}$ of VX-770 and its derivatives at $27^{\circ} \mathrm{C}$ for 24 hours. There was a significant correlation, suggesting that the higher the lipophilicity of the drug derivative may correlate to less cell surface expression of F508delCFTR ( $n=4$ for all derivatives). Results were analyzed by linear regression and Spearman's correlation $(r=-0.69$ and $P=0.047)$. (C) Comparisons of band $\mathrm{C}$ relative to band $\mathrm{C}$ and band $\mathrm{B}(\mathrm{C}+\mathrm{B})$ of F508del-CFTR chronically treated with DMSO, $10 \mu \mathrm{M}$ VX-770 and $10 \mu \mathrm{M}$ SE-03 at $27^{\circ} \mathrm{C}$ for 24 hours. Densitometry analyses show that treatment with $10 \mu \mathrm{M}$ VX-770 significantly decreased band C relative to band C+B of F508del-CFTR, whereas treatment with $10 \mu \mathrm{M}$ SE-03 did not compared with DMSO ( $n=5$ for all conditions). Results are presented as mean \pm S.D. and analyzed by one-way ANOVA and Dunnett's post hoc test (**P $<0.01$ compared with vehicle). (D) FLiPR channel function studies of VX-809-rescued F508del-CFTR stimulated (Stim.) with vehicle (labeled as "a"), $1 \mu \mathrm{M}$ cAMP agonist, forskolin (Fsk) (labeled as "b"), $1 \mu \mathrm{M}$ Fsk and $10 \mu \mathrm{M}$ VX-770 (labeled as "c"), or $1 \mu \mathrm{M}$

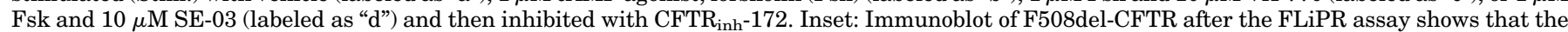
level of band $\mathrm{C}$ in each condition was relatively similar compared with calnexin (CNX) as a loading control. (E) Quantification of the maximum peak of Fsk stimulation in the FLiPR assay relative to baseline and DMSO shows that acute treatment with $10 \mu \mathrm{M}$ VX-770 and $10 \mu \mathrm{M}$ SE-03 with $1 \mu \mathrm{M}$ Fsk significantly potentiated CFTR chloride flux compared with $1 \mu \mathrm{M}$ Fsk alone (DMSO, $n=3$ for all conditions, average of eight wells per replicate). Results are presented as mean \pm S.D. and analyzed by one-way ANOVA and Dunnett's post hoc test $(* P<0.05 ; * * P<0.01$ compared with $1 \mu \mathrm{M}$ Fsk $)$.

and DOPC/cholesterol/sphingomyelin (bSM) bathed in a HEPES buffer (Simons and Toomre, 2000; Calder and Yaqoob, 2007). Two distinct bilayers were tested for the effect of VX-770 with three to six fields of view analyzed per bilayer (Fig. 5, A and B). Order parameter $<\mathrm{P}_{2}>$ measurements and the histogram movies demonstrated that $10 \mu \mathrm{M}$ VX-770 immediately increased the bilayer fluidity as well as reorganized subdomains within the DOPC/bSM/cholesterol supported lipid bilayers. The histograms were determined from the selected field of view and that same field of view was used to track the dynamic changes in the bilayer upon VX-770 addition. As such, our interpretations as to the effect of these agents on membrane stability and structure are drawn from relative changes in the individual histogram characteristics for the chosen field of view. From that perspective, we can clearly see that there is a clear shift to a higher mean $\left\langle\mathrm{P}_{2}\right\rangle$ value and a tighter distribution upon VX-770, which is strongly suggestive of increased membrane fluidity. This interpretation is certainly bolstered by the before- and afterVX-770 addition, which shows significant changes in the bilayer domain structures. Conversely, the relative decrease and broadening of the distribution of $\left\langle\mathrm{P}_{2}\right\rangle$ values (i.e., emergence of a second lower $<\mathrm{P}_{2}>$ peak) upon SE-03 addition can only be interpreted at this point as evidence of a greater extent of membrane reorganization. Intriguingly, the extent of subdomain restructuring due to SE-03 addition appears to be significantly less than in the VX-770 case, as is evidenced by the relatively minor changes in bilayer morphology 
$10 \mu \mathrm{M} V X-770$

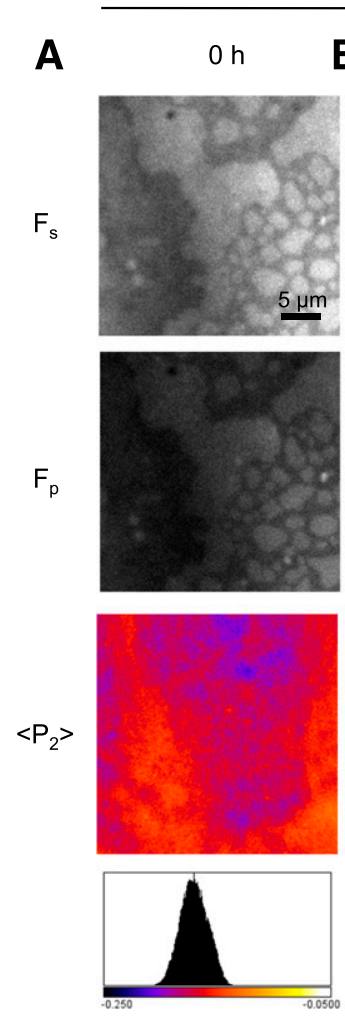

$-0.171 \pm 0.0120$
B
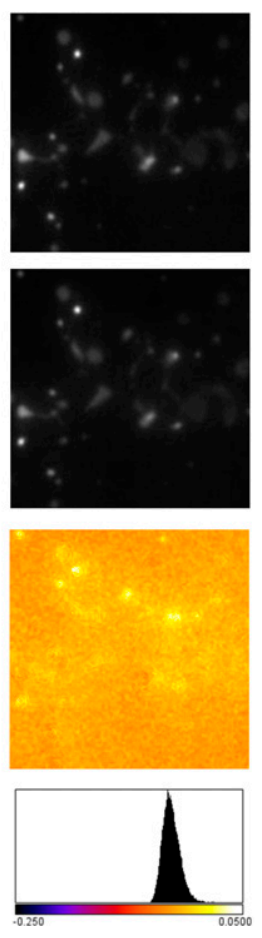

$-0.0438 \pm 0.0122$
$10 \mu \mathrm{M}$ SE-03
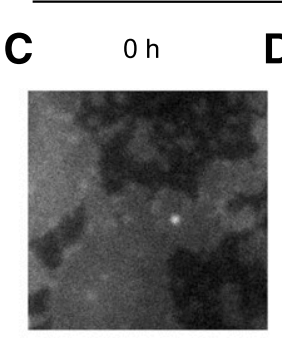

D $1 \mathrm{~h}$
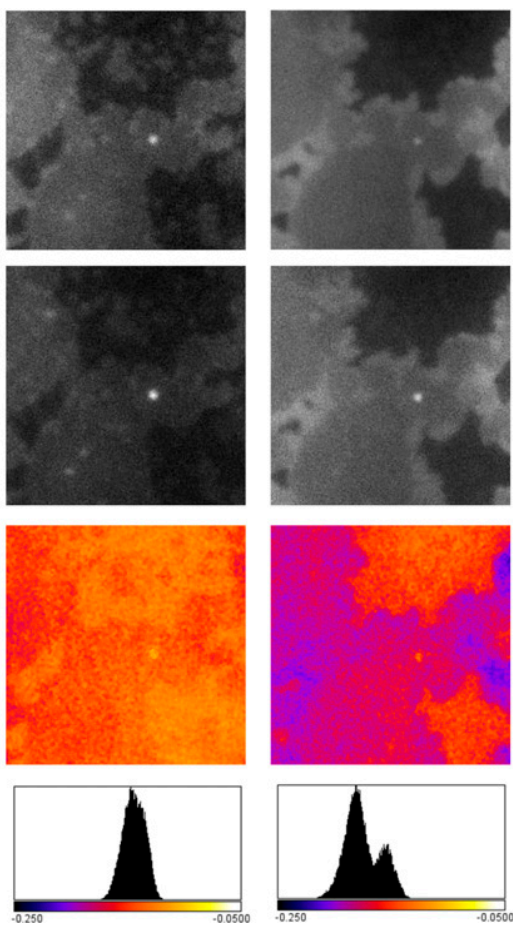

$-0.144 \pm 0.00972$
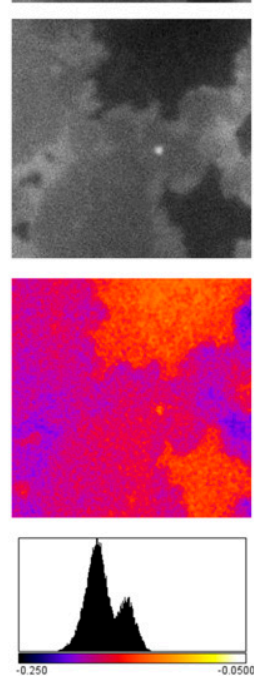

$-0.175 \pm 0.0157$
Fig. 5. Suprapharmacological concentration of VX-770 appears to increase membrane fluidity and reorganize the bilayer. Representative pTIRF images of a $30 \times 30 \mu \mathrm{m}$ section of a DOPC/bSM/cholesterol lipid raft-like model prior to addition of (A) $10 \mu \mathrm{M}$ VX-770 and (C) $10 \mu \mathrm{M}$ SE-03. pTIRF images at 1 hour incubation with (B) $10 \mu \mathrm{M}$ VX-770 and (D) $10 \mu \mathrm{M}$ SE-03. First row represents the pTIRF images obtained by substrate parallel excitation $\left(\mathrm{F}_{\mathrm{s}}\right)$ and the second row represents the pTIRF images obtained by perpendicular excitation $\left(\mathrm{F}_{\mathrm{p}}\right)$ of the DiI- $\mathrm{C}_{18}$ fluorescent dye. Third row represents the same images colored by the order parameter $\left.<\mathrm{P}_{2}\right\rangle$. The last row represents the histogram summarizing the range of $\left\langle\mathrm{P}_{2}\right\rangle$, with the mean value and margin of error indicated at the bottom. Two bilayers were studied per condition with three to six fields analyzed per bilayer.
(Fig. 5, C and D), supporting the claim the VX-770 effects are mediated at least in part by its relative lipophilicity.

\section{Discussion}

The current studies provide a potential explanation for the concentration-dependent, destabilizing effects of chronic exposure to VX-770 on VX-809-rescued F508del-CFTR protein (Cholon et al., 2014; Veit et al., 2014; Matthes et al., 2016). At the concentrations of VX-770 that reduce F508del-CFTR protein stability, we also observed reduction of the abundance of other membrane proteins (SLC26A3, SLC26A9, and SLC6A14), pointing to its lack of specificity. From our studies of model lipid rafts, we deduce that this negative effect of high concentrations of VX-770 is related to its lipophilicity and disruption of these membrane structures.

Our biochemical studies showed that the concentration of VX-770 $(10 \mu \mathrm{M})$ that reduced VX-809-mediated augmentation in F508del-CFTR processing also reversed VX-809-mediated enhancement of the interaction between the intracellular loop conferred by MSD2 (ICL4) and NBD1. As in previous studies (He et al., 2008; Loo et al., 2008; Serohijos et al., 2008; Aleksandrov et al., 2010; Loo and Clarke, 2011; Chin et al., 2017), we probed the interaction at this interface in the context of a cys-less variant of F508del-CFTR protein harboring cysteine residues engineered in ICL4 (A1067C) and NBD1 (V510C). In our experimental system, we could not detect VX-809-induced maturation and processing of the V510C/A1067C cys-less variant of F508del-CFTR protein in immunoblots. However, VX-809-induced interface assembly in the immature, core glycosylated band B of the protein was detectable as a unique cross-linked band $(*)$ and the relative abundance of this band was reduced in the presence of $10 \mu \mathrm{M}$ VX-770 but not in the presence of $100 \mathrm{nM}$ VX-770. Hence, we interpret the destabilizing effects of VX-770 at this concentration to be due to inhibition of the positive effects of VX-809 at this interface. However, as discussed in the subsequent paragraphs, this effect is likely not specific to this interface or even the mutant CFTR protein.

The same concentration of VX-770 that induced destabilizing effects on F508del-CFTR assembly also reduced the steadystate abundance of the three SLC proteins tested. We chose these proteins because they are known to be localized in the same tissues and membranes as CFTR and modify epithelial transport function (Ko et al., 2004; Mount and Romero, 2004; Sun et al., 2012). Hence, any nonspecific effect of VX-770 on these membrane proteins may have a potential to modify disease and/or therapeutic response to ORKAMBI. Of these proteins, SLC26A3 was the most sensitive to the destabilizing effect of VX-770, with a significant decrease in protein abundance apparent (at least in the HEK-293 expression system) at $1 \mu \mathrm{M}$, the serum concentration reported by Vertex Pharmaceuticals (https://pi.vrtx.com/files/uspi_ivacaftor.pdf). Previous studies have shown that loss of function mutations of SLC26A3 caused a rare disease known as congenital chloride-losing diarrhea via impaired chloride absorption and fluid/bicarbonate secretion (Höglund et al., 1996). Intestinal inflammation, which is common in diarrhea, has been found to further 
reduce SLC26A3 expression through direct binding to NF- $\kappa \mathrm{B}$ (Kumar et al., 2017). Hence, it is possible that the destabilizing effect of VX-770 that we observed on SLC26A3 may modify the ORKAMBI response in the intestinal tissues.

SLC26A9 confers an apical anion channel, and this function augments the cAMP-regulated channel activity of CFTR in the non-CF airway epithelium and in the epithelium of patients bearing gating mutations such as G551D (Bertrand et al., 2009, 2017). Strug et al. (2016) showed that polymorphisms in the SLC26A9 gene associate with the response of patients bearing G551D to VX-770 and interpreted these results to suggest that functional expression of SLC26A9 enhances the therapeutic efficacy of VX-770 in this cohort of patients. Therefore, VX-770 in patient tissues is not impairing the positive effect of SLC26A9 on the potentiation of the G551D channel. On the other hand, the misfolded F508del-CFTR protein interacts with and prevents SLC26A9 trafficking to the apical surface (Bertrand et al., 2009, 2017). Correction of F508del-CFTR by VX-809 is thought to promote SLC26A9 trafficking to the cell surface indirectly (Bertrand et al., 2009, 2017). In vitro studies of bronchial explant tissue from F508del homozygotes suggest that the magnitude of pharmacological correction by VX-809 is also augmented by SLC26A9 expression (Strug et al., 2016). Hence, the results of the current study suggest that the possible deleterious effect of chronic VX-770 on SLC26A9 protein stability should be further studied given its importance in modifying the pharmacological rescue conferred by this therapy.

SLC6A14 is an apical amino acid transporter, and we have shown that it functions to remove nutrient amino acids from the surface of respiratory epithelial cells (Di Paola et al., 2017). This function is associated with reduced adherence of Pseudomonas aeruginosa to the surface of respiratory epithelial cells (Di Paola et al., 2017). We speculate that reduced surface stability of SLC6A14 induced by high concentrations of VX-770 would potentially enhance $P$. aeruginosa attachment and biofilm formation and we are testing this hypothesis.

We propose that the destabilizing effect of VX-770 is related to its lipophilic property. Interestingly, treatment of F508delCFTR with a panel of VX-770 derivatives showed that the degree of VX-770 lipophilicity is correlated to its destabilizing effect. SE-03 lacks two tert-butyl groups on the phenol ring (Supplemental Fig. 2) and is less lipophilic than VX-770 (the $\log P$ value of SE-03 is 2.67, whereas it is 5.76 for VX-770), yet it retains activity as a potentiator. Interestingly, SE-03 does not reduce F508del-CFTR protein stability even at the high concentration of $10 \mu \mathrm{M}$. Biophysical studies of a lipid raft model showed that unlike VX-770, SE-03 addition did not disperse lipid rafts comprised of cholesterol and sphingomyelin. Together, these findings support the hypothesis that the nonspecific effects of VX-770 on membrane protein stability are mediated through VX-770 interaction with and disruption of lipid rafts, a membrane organizing structure known to facilitate protein interactions and signaling (Bi et al., 2001; Patel et al., 2008; Steinberg, 2008; Abu-Arish et al., 2015).

The nonspecific effect of chronic VX-770 on the steady-state abundance of F508del-CFTR, other membrane proteins, and lipid rafts was observed at the suprapharmacological concentration of $10 \mu \mathrm{M}$ VX-770. According to Matthes et al. (2016), a peak free plasma concentration of VX-770 is $1.5-8.5 \mathrm{nM}$, considerably lower than other estimates and the concentration at which we observed the nonspecific effects of VX-770 (Hanrahan et al., 2017). According to the drug data sheet provided by Vertex, after a single recommended dose of $150 \mathrm{mg}$ administered after a meal, the peak plasma concentration reaches $768 \mathrm{ng} / \mathrm{ml}$ or 1 to $2 \mu \mathrm{M}$ (https://pi.vrtx.com/ files/uspi_ivacaftor.pdf). This value is an order of magnitude less than the concentration $(10 \mu \mathrm{M})$ that caused significant loss of CFTR and other membrane proteins (including SLC26A9) in the in vitro studies of this paper. However, according to a previous study (Trittler and Hug, 2014), there was a large range in serum concentrations of VX-770 in different patients, ranging from 400 to $3000 \mathrm{ng} / \mathrm{ml}$, with the upper limit approaching $10 \mu \mathrm{M}$. Importantly, the concentration of VX-770 in the tissues of CF patients treated with VX-770 is uncertain. According to the European Medicine Agency (EMA/473279/2012), VX-770 accumulates in lung and tracheal epithelial tissues relative to plasma in rat studies (http://www.ema.europa.eu/docs/en_GB/document_ library/EPAR_-_Public_assessment_report/human/002494/ WC500130766.pdf). Hence, it remains unknown if tissue concentrations could approach the higher concentration of $10 \mu \mathrm{M}$ shown to exert nonspecific, destabilizing effects.

It is important to acknowledge that VX-770 exerts a beneficial effect overall when given as a monotherapy to individuals bearing gating mutations in CFTR. However, our studies suggest that future clinical studies are required to monitor offtarget effects in this population taking this monotherapy continuously over their lifetime. Finally, it remains possible that the variable clinical efficacy of ORKAMBI in the F508del patient population is partially associated with variation in its tissue accumulation among patients and/or off-target effects of VX-770 on other membrane proteins variably expressed in different patients.

\section{Acknowledgments}

We thank Dr. David Clarke for providing the V510C, A1067C and V510C/A1067C cys-less variants of wt-CFTR. We thank Dr. Reinhart Reithmeier and OriGene for providing the SLC constructs. We thank Dr. Steven Molinski for the helpful discussions on these studies. We thank Dr. Jacqueline McCormack for help in editing the manuscript.

\section{Authorship Contributions}

Participated in research design: Chin, Hung, Won, Wu, Yip, Bear. Conducted experiments: Chin, Hung, Won, Wu.

Contributed new reagents or analytic tools: Ahmadi, Yang, Elmallah, Toutah, Hamilton, Young, Viirre, Yip.

Performed data analysis: Chin, Hung, Won, Wu, Bear.

Wrote or contributed to the writing of the manuscript: Chin, Hung, Won, Young, Viirre, Yip, Bear.

\section{References}

Abu-Arish A, Pandzic E, Goepp J, Matthes E, Hanrahan JW, and Wiseman PW (2015) Cholesterol modulates CFTR confinement in the plasma membrane of primary epithelial cells. Biophys $J$ 109:85-94.

Aleksandrov AA, Kota P, Aleksandrov LA, He L, Jensen T, Cui L, Gentzsch M, Dokholyan NV, and Riordan JR (2010) Regulatory insertion removal restores maturation, stability and function of DeltaF508 CFTR. J Mol Biol 401:194-210.

Anagnostopoulou P, Riederer B, Duerr J, Michel S, Binia A, Agrawal R, Liu X, Kalitzki K, Xiao F, Chen M, et al. (2012) SLC26A9-mediated chloride secretion prevents mucus obstruction in airway inflammation. J Clin Invest 122:3629-3634. Avella M, Loriol C, Boulukos K, Borgese F, and Ehrenfeld J (2011) SLC26A9 stimulates CFTR expression and function in human bronchial cell lines. J Cell Physiol 226:212-223.

Baroni D, Zegarra-Moran O, Svensson A, and Moran O (2014) Direct interaction of a CFTR potentiator and a CFTR corrector with phospholipid bilayers. Eur Biophys $J$ 43:341-346.

Bertrand CA, Mitra S, Mishra SK, Wang X, Zhao Y, Pilewski JM, Madden DR, and Frizzell RA (2017) The CFTR trafficking mutation F508del inhibits the constitutive activity of SLC26A9. Am J Physiol Lung Cell Mol Physiol 312:L912-L925. 
Bertrand CA, Zhang R, Pilewski JM, and Frizzell RA (2009) SLC26A9 is a constitutively active, CFTR-regulated anion conductance in human bronchial epithelia. $J$ Gen Physiol 133:421-438.

Bi K, Tanaka Y, Coudronniere N, Sugie K, Hong S, van Stipdonk MJ, and Altman A (2001) Antigen-induced translocation of PKC-theta to membrane rafts is required for T cell activation. Nat Immunol 2:556-563.

Boyle MP, Bell SC, Konstan MW, McColley SA, Rowe SM, Rietschel E, Huang X, Waltz D, Patel NR, and Rodman D; VX09-809-102 study group (2014) A CFTR corrector (lumacaftor) and a CFTR potentiator (ivacaftor) for treatment of patients with cystic fibrosis who have a phe508del CFTR mutation: a phase 2 randomised controlled trial. Lancet Respir Med 2:527-538.

Calder PC and Yaqoob P (2007) Lipid rafts-composition, characterization, and controversies. J Nutr 137:545-547.

Chang MH, Plata C, Sindic A, Ranatunga WK, Chen AP, Zandi-Nejad K, Chan KW, Thompson J, Mount DB, and Romero MF (2009) Slc26a9 is inhibited by the R-region of the cystic fibrosis transmembrane conductance regulator via the STAS domain. J Biol Chem 284:28306-28318.

Chin S, Yang D, Miles AJ, Eckford PD, Molinski S, Wallace BA, and Bear CE (2017) Attenuation of phosphorylation-dependent activation of cystic fibrosis transmembrane conductance regulator (CFTR) by disease-causing mutations at the transmission interface. J Biol Chem 292:1988-1999.

Cholon DM, Quinney NL, Fulcher ML, Esther CR Jr, Das J, Dokholyan NV, Randell SH, Boucher RC, and Gentzsch M (2014) Potentiator ivacaftor abrogates pharmacological correction of $\Delta$ F508 CFTR in cystic fibrosis. Sci Transl Med 6:246ra96.

Corvol H, Blackman SM, Boëlle PY, Gallins PJ, Pace RG, Stonebraker JR, Accurso FJ, Clement A, Collaco JM, Dang H, et al. (2015) Genome-wide association metaanalysis identifies five modifier loci of lung disease severity in cystic fibrosis. Nat Commun 6:8382.

Cutting GR (2015) Cystic fibrosis genetics: from molecular understanding to clinical application. Nat Rev Genet 16:45-56.

Di Paola M, Park AJ, Ahmadi S, Roach EJ, Wu YS, Struder-Kypke M, Lam JS, Bear CE, and Khursigara CM (2017) SLC6A14 is a genetic modifier of cystic fibrosis that regulates Pseudomonas aeruginosa attachment to human bronchial epithelial cells. MBio 8:e2073-17

Eckford PD, Li C, Ramjeesingh M, and Bear CE (2012) Cystic fibrosis transmembrane conductance regulator (CFTR) potentiator VX-770 (ivacaftor) opens the defective channel gate of mutant CFTR in a phosphorylation-dependent but ATPindependent manner. $J$ Biol Chem 287:36639-36649.

Gupta N, Prasad PD, Ghamande S, Moore-Martin P, Herdman AV, Martindale RG, Podolsky R, Mager S, Ganapathy ME, and Ganapathy V (2006) Up-regulation of the amino acid transporter $\operatorname{ATB}(0,+)(\mathrm{SLC6A14})$ in carcinoma of the cervix. Gynecol Oncol 100:8-13.

Hanrahan JW, Matthes E, Carlile G, and Thomas DY (2017) Corrector combination therapies for F508del-CFTR. Curr Opin Pharmacol 34:105-111.

He L, Aleksandrov AA, Serohijos AW, Hegedus T, Aleksandrov LA, Cui L, Dokholyan NV, and Riordan JR (2008) Multiple membrane-cytoplasmic domain contacts in the cystic fibrosis transmembrane conductance regulator (CFTR) mediate regulation of channel gating. J Biol Chem 283:26383-26390.

He L, Kota P, Aleksandrov AA, Cui L, Jensen T, Dokholyan NV, and Riordan JR (2013) Correctors of $\triangle$ F508 CFTR restore global conformational maturation with out thermally stabilizing the mutant protein. FASEB J 27:536-545.

Höglund P, Haila S, Socha J, Tomaszewski L, Saarialho-Kere U, KarjalainenLindsberg ML, Airola K, Holmberg C, de la Chapelle A, and Kere J (1996) Mutations of the down-regulated in adenoma (DRA) gene cause congenital chloride diarrhoea. Nat Genet 14:316-319.

Jih KY and Hwang TC (2013) Vx-770 potentiates CFTR function by promoting decoupling between the gating cycle and ATP hydrolysis cycle. Proc Natl Acad Sci USA 110:4404-4409.

Karunakaran S, Ramachandran S, Coothankandaswamy V, Elangovan S, Babu E, Periyasamy-Thandavan S, Gurav A, Gnanaprakasam JP, Singh N, Schoenlein PV, et al. (2011) SLC6A14 (ATB0,+) protein, a highly concentrative and broad specific amino acid transporter, is a novel and effective drug target for treatment of estrogen receptor-positive breast cancer. J Biol Chem 286:31830-31838.

Ko SB, Zeng W, Dorwart MR, Luo X, Kim KH, Millen L, Goto H, Naruse S, Soyombo A, Thomas PJ, et al. (2004) Gating of CFTR by the STAS domain of SLC26 transporters. Nat Cell Biol 6:343-350.

Kumar A, Chatterjee I, Gujral T, Alakkam A, Coffing H, Anbazhagan AN, Borthakur A, Saksena S, Gill RK, Alrefai WA, et al. (2017) Activation of nuclear factor-кB by tumor necrosis factor in intestinal epithelial cells and mouse intestinal epithelia reduces expression of the chloride transporter SLC26A3. Gastroenterology 153: 1338-1350.e3.

Li J, Xia F, and Reithmeier RA (2014a) N-glycosylation and topology of the human SLC26 family of anion transport membrane proteins. Am J Physiol Cell Physiol 306:C943-C960

Li W, Soave D, Miller MR, Keenan K, Lin F, Gong J, Chiang T, Stephenson AL, Durie P, Rommens J, et al. (2014b) Unraveling the complex genetic model for cystic fibrosis: pleiotropic effects of modifier genes on early cystic fibrosis-related morbidities. Hum Genet 133:151-161.

Loo TW, Bartlett MC, and Clarke DM (2008) Processing mutations disrupt interactions between the nucleotide binding and transmembrane domains of P-glycoprotein and the cystic fibrosis transmembrane conductance regulator (CFTR). J Biol Chem 283:28190-28197.

Loo TW, Bartlett MC, and Clarke DM (2013) Corrector VX-809 stabilizes the first transmembrane domain of CFTR. Biochem Pharmacol 86:612-619.

Loo TW and Clarke DM (2006) Using a cysteine-less mutant to provide insight into the structure and mechanism of CFTR [published correction appears in J Physiol (2006) 573:569]. J Physiol 572:312.
Loo TW and Clarke DM (2011) Repair of CFTR folding defects with correctors that function as pharmacological chaperones. Methods Mol Biol 741:23-37.

Matthes E, Goepp J, Carlile GW, Luo Y, Dejgaard K, Billet A, Robert R, Thomas DY, and Hanrahan JW (2016) Low free drug concentration prevents inhibition of F508del CFTR functional expression by the potentiator VX-770 (ivacaftor). $\mathrm{Br}$ $J$ Pharmacol 173:459-470.

Mendoza JL, Schmidt A, Li Q, Nuvaga E, Barrett T, Bridges RJ, Feranchak AP, Brautigam CA, and Thomas PJ (2012) Requirements for efficient correction of $\Delta$ F508 CFTR revealed by analyses of evolved sequences. Cell 148:164-174.

Molinski SV, Ahmadi S, Hung M, and Bear CE (2015) Facilitating structure-function studies of CFTR modulator sites with efficiencies in mutagenesis and functional screening. J Biomol Screen 20:1204-1217.

Mount DB and Romero MF (2004) The SLC26 gene family of multifunctional anion exchangers. Pflugers Arch 447:710-721.

Okiyoneda T, Veit G, Dekkers JF, Bagdany M, Soya N, Xu H, Roldan A, Verkman AS, Kurth M, Simon A, et al. (2013) Mechanism-based corrector combination restores $\triangle$ F508-CFTR folding and function. Nat Chem Biol 9:444-454

Oreopoulos J, Epand RF, Epand RM, and Yip CM (2010) Peptide-induced domain formation in supported lipid bilayers: direct evidence by combined atomic force and polarized total internal reflection fluorescence microscopy. Biophys $J$ 98: $815-823$

Oreopoulos J and Yip CM (2009) Probing membrane order and topography in supported lipid bilayers by combined polarized total internal reflection fluorescenceatomic force microscopy. Biophys J 96:1970-1984.

Patel HH, Murray F, and Insel PA (2008) G-protein-coupled receptor-signaling components in membrane raft and caveolae microdomains. Handb Exp Pharmacol (186):167-184

Pedemonte N, Sonawane ND, Taddei A, Hu J, Zegarra-Moran O, Suen YF, Robins LI Dicus CW, Willenbring D, Nantz MH, et al. (2005) Phenylglycine and sulfonamide correctors of defective delta F508 and G551D cystic fibrosis transmembrane conductance regulator chloride-channel gating. Mol Pharmacol 67: 1797-1807.

Rabeh WM, Bossard F, Xu H, Okiyoneda T, Bagdany M, Mulvihill CM, Du K, di Bernardo S, Liu Y, Konermann L, et al. (2012) Correction of both NBD1 energetics and domain interface is required to restore $\triangle$ F508 CFTR folding and function. Cell 148:150-163.

Ren HY, Grove DE, De La Rosa O, Houck SA, Sopha P, Van Goor F, Hoffman BJ, and Cyr DM (2013) VX-809 corrects folding defects in cystic fibrosis transmembrane conductance regulator protein through action on membrane-spanning domain 1. Mol Biol Cell 24:3016-3024.

Serohijos AW, Hegedus T, Aleksandrov AA, He L, Cui L, Dokholyan NV, and Riordan JR (2008) Phenylalanine-508 mediates a cytoplasmic-membrane domain contact in the CFTR 3D structure crucial to assembly and channel function. Proc Natl Acad Sci USA 105:3256-3261.

Simons K and Toomre D (2000) Lipid rafts and signal transduction. Nat Rev Mol Cell Biol 1:31-39.

Steinberg SF (2008) Structural basis of protein kinase C isoform function. Physiol Rev 88:1341-1378.

Strug LJ, Gonska T, He G, Keenan K, Ip W, Boëlle PY, Lin F, Panjwani N, Gong J, $\mathrm{Li} \mathrm{W}$, et al. (2016) Cystic fibrosis gene modifier SLC26A9 modulates airway response to CFTR-directed therapeutics. Hum Mol Genet 25:4590-4600.

Sun L, Rommens JM, Corvol H, Li W, Li X, Chiang TA, Lin F, Dorfman R, Busson PF, Parekh RV, et al. (2012) Multiple apical plasma membrane constituents are associated with susceptibility to meconium ileus in individuals with cystic fibrosis. Nat Genet 44:562-569.

Trittler R and Hug M (2014) PKP-017 monitoring of ivacaftor serum levels. Eur $J$ Hosp Pharm Sci Pract 21 (Suppl 1):A143-A144.

Van Goor F, Hadida S, Grootenhuis PD, Burton B, Cao D, Neuberger T, Turnbull A, Singh A, Joubran J, Hazlewood A, et al. (2009) Rescue of CF airway epithelial cell function in vitro by a CFTR potentiator, VX-770. Proc Natl Acad Sci USA 106 18825-18830.

Van Goor F, Hadida S, Grootenhuis PD, Burton B, Stack JH, Straley KS, Decker CJ, Miller M, McCartney J, Olson ER, et al. (2011) Correction of the F508del-CFTR protein processing defect in vitro by the investigational drug VX-809. Proc Natl Acad Sci USA 108:18843-18848.

Veit G, Avramescu RG, Perdomo D, Phuan PW, Bagdany M, Apaja PM, Borot F, Szollosi D, Wu YS, Finkbeiner WE, et al. (2014) Some gating potentiators, including VX-770, diminish $\Delta$ F508-CFTR functional expression. Sci Transl Med 6 $246 \mathrm{ra} 97$.

Walsh P, Vanderlee G, Yau J, Campeau J, Sim VL, Yip CM, and Sharpe S (2014) The mechanism of membrane disruption by cytotoxic amyloid oligomers formed by prion protein(106-126) is dependent on bilayer composition. J Biol Chem $\mathbf{2 8 9}$ : 10419-10430.

Wang Y, Loo TW, Bartlett MC, and Clarke DM (2007) Correctors promote maturation of cystic fibrosis transmembrane conductance regulator (CFTR)-processing mutants by binding to the protein. J Biol Chem 282:33247-33251.

Xu J, Henriksnäs J, Barone S, Witte D, Shull GE, Forte JG, Holm L, and Soleimani M (2005) SLC26A9 is expressed in gastric surface epithelial cells, mediates Cl-/ HCO3- exchange, and is inhibited by NH4+. Am J Physiol Cell Physiol 289 C493-C505.

Address correspondence to: Dr. Christine E. Bear, Peter Gilgan Centre for Research and Learning, Department of Molecular Medicine, Hospital for Sick Children, 686 Bay Street, Room 209420 U-West, Toronto, ON, M5G 0A4. E-mail: bear@sickkids.ca 